\title{
Editorial for the Special Issue "New Advances on Sub-Pixel Processing: Unmixing and Mapping Methods"
}

\author{
Addisson Salazar*(D), Luis Vergara (D) and Gonzalo Safont (D) \\ Institute of Telecommunications and Multimedia Applications, Universitat Politècnica de València, \\ Camino de Vera s/n, 46022 Valencia, Spain; Ivergara@dcom.upv.es (L.V.); gonsaar@upvnet.upv.es (G.S.) \\ * Correspondence: asalazar@dcom.upv.es
}

check for updates

Citation: Salazar, A.; Vergara, L.; Safont, G. Editorial for the Special Issue “New Advances on Sub-Pixel Processing: Unmixing and Mapping Methods". Remote Sens. 2021, 13, 3807 https://doi.org/10.3390/rs13193807

Received: 6 September 2021

Accepted: 13 September 2021

Published: 23 September 2021

Publisher's Note: MDPI stays neutral with regard to jurisdictional claims in published maps and institutional affiliations.

Copyright: (c) 2021 by the authors. Licensee MDPI, Basel, Switzerland. This article is an open access article distributed under the terms and conditions of the Creative Commons Attribution (CC BY) license (https:// creativecommons.org/licenses/by/ $4.0 /$ )
Innovative remote sensing image processing techniques have been progressively studied due to the increasing availability of remote sensing images, powerful techniques of data analysis, and computational power. Sub-pixel processing has emerged as an intense area of research considering the possibility of a pixel to belong to different classes in an image segmentation context. This is especially relevant in remote sensing, where different macroscopic or microscopic components could appear to contribute to every pixel. Thus, sub-pixel segmentation can increase the resolution of the original images, which is limited by the sensorial systems and several measurement effects. Two main problems are identified in sub-pixel processing: unmixing and mapping. Unmixing refers to the separation of the components contributing to the pixel information (typically a pixel signature obtained from hyperspectral images) to build abundance maps describing the proportion of every component in a given pixel. Mapping is the distribution inside a pixel of its labeled sub-pixels, consistent with the abundance maps.

In this Special Issue, several stages of sub-pixel image processing are approached incorporating advanced techniques such as neural networks, deep learning, and probabilistic non-Gaussian mixture models. This Special Issue consists of nine research papers [1-9]. All the methods proposed in the papers were validated using real hyperspectral data and benchmarked with state-of-the-art methods, thus comprehensively demonstrating the theoretical and practical contributions of the papers. The first paper contains a sub-pixel mapping model based on spatial regularization using the total variation and a dictionary of pre-learned spatial patterns for improving the class sub-pixel spatial localization [1]. Thus, the sub-pixel mapping problem is transformed into a regularization problem integrating the isotropic total variation minimization as a prior model applied to the abundance maps.

With the development of satellite technology, the quality of remote sensing images is increasing dramatically. Retrieving similar remote sensing images from large-scale datasets is very important and demanding. To reduce the retrieval complexity of remote sensing image retrieval, a new hash learning method is proposed in [2]. This method generates compact hash codes using low-rankness constraints on the projection matrix to exploit its global structure and hypergraphs to capture the high-order relationship among the data.

Three papers of this Special Issue are focused on the estimation of endmembers in the unmixing of hyperspectral images. Thus, ref. [3] introduces a new hierarchical agglomerative clustering algorithm based on probabilistic non-Gaussian mixture models to estimate the number of endmembers. This method obtains a hierarchy of image partitions and selects a partition using a validation index; the estimated number of materials or substances that are present in the scene is set to the number of clusters of the selected partition. A challenging issue when unmixing hyperspectral data is the variability that is associated with each endmember. In order to handle this problem, an unmixing model that not only extracts and clusters the prototypal endmember spectra but also estimates the abundances of each member is proposed in [4]. The prototypal endmember spectral variability is modeled as a conical hull defined by extremal pixels of the dataset. Another 
method to deal with endmember variability and selection for sub-pixel mapping of surface water (water fraction maps) is included in [5]. A multi-index threshold extraction method is applied to select the endmembers, and endmember variability considers typical and neighboring endmembers in multiple endmember spectral mixture analysis.

Neural networks have provided a set of powerful tools that have allowed remote sensing applications to be boosted. Thus, two neural-based methods for sub-pixel urban inundation mapping and super-resolution mapping have been developed in [6,7], respectively. Urban flooding is one of the most costly and destructive natural hazards worldwide. The method proposed in [6] implements Elman neural networks coupled with spatial attraction models to improve the accuracy of sub-pixel urban inundation mapping. The method proposed in [7] develops a convolutional neural network $(\mathrm{CNN})$ for super-resolution land cover mapping that allows fine-scale land cover maps from coarse remote sensing images to be obtained. The implemented CNN captures the spatial characteristics of geographic objects by modeling the nonlinear relationship between coarse remote sensing images and fine-scale land cover maps. On the other hand, super-resolution mapping is also studied in [8] for burned area mapping based on space-temperature information. The proposed method uses the random walker algorithm to characterize the space element; the temperature element is derived by calculating the normalized burn ratio; and both elements are merged in an objective function that is handled by using the particle swarm optimization algorithm to derive the burned area mapping results.

Finally, spatio-temporal sub-pixel land cover mapping is approached in [9]. The objective is to obtain both a fine spatial and temporal resolution using a few fine-spatial resolution maps and a time series of coarse-spatial resolution remote sensing images. The method uses ancillary data such as the pixel spectral similarity to enhance the quality of the fine-spatial resolution maps, thus improving the overall accuracy and prediction of the changed pixels.

Funding: This research received no external funding.

Acknowledgments: The Guest Editors of this Special Issue would like to thank all authors who have contributed to this volume for sharing their scientific results and experiences. We would also like to thank the journal editorial team and reviewers for conducting the review process.

Conflicts of Interest: The authors declare no conflict of interest.

\section{References}

1. Msellmi, B.; Picone, D.; Ben Rabah, Z.; Dalla Mura, M.; Farah, I.R. Sub-Pixel Mapping Model Based on Total Variation Regularization and Learned Spatial Dictionary. Remote Sens. 2021, 13, 190. [CrossRef]

2. Kong, L.; Sun, Q.; Mukherjee, M.; Lloret, J. Low-Rank Hypergraph Hashing for Large-Scale Remote Sensing Image Retrieval. Remote Sens. 2020, 12, 1164. [CrossRef]

3. Prades, J.; Safont, G.; Salazar, A.; Vergara, L. Estimation of the Number of Endmembers in Hyperspectral Images Using Agglomerative Clustering. Remote Sens. 2020, 12, 3585. [CrossRef]

4. Uezato, T.; Fauvel, M.; Dobigeon, N. Hierarchical Sparse Nonnegative Matrix Factorization for Hyperspectral Unmixing with Spectral Variability. Remote Sens. 2020, 12, 2326. [CrossRef]

5. Liu, C.; Shi, J.; Liu, X.; Shi, Z.; Zhu, J. Subpixel Mapping of Surface Water in the Tibetan Plateau with MODIS Data. Remote Sens. 2020, 12, 1154. [CrossRef]

6. Li, L.; Chen, Y.; Xu, T.; Meng, L.; Huang, C.; Shi, K. Spatial Attraction Models Coupled with Elman Neural Networks for Enhancing Sub-Pixel Urban Inundation Mapping. Remote Sens. 2020, 12, 2068. [CrossRef]

7. Jia, Y.; Ge, Y.; Chen, Y.; Li, S.; Heuvelink, G.B.M.; Ling, F. Super-Resolution Land Cover Mapping Based on the Convolutional Neural Network. Remote Sens. 2019, 11, 1815. [CrossRef]

8. Wang, P.; Zhang, L.; Zhang, G.; Jin, B.; Leung, H. Multispectral Image Super-Resolution Burned-Area Mapping Based on Space-Temperature Information. Remote Sens. 2019, 11, 2695. [CrossRef]

9. Li, X.; Chen, R.; Foody, G.M.; Wang, L.; Yang, X.; Du, Y.; Ling, F. Spatio-Temporal Sub-Pixel Land Cover Mapping of Remote Sensing Imagery Using Spatial Distribution Information From Same-Class Pixels. Remote Sens. 2020, 12, 503. [CrossRef] 